\title{
LA LENGUA DE SIGNOS COMO RECURSO INCLUSIVO EN EL SISTEMA EDUCATIVO ESPAÑOL
}

Sign Language as an Inclusive Resource in the Spanish Educational System

\section{Silvia Cristina Benito-Moreno ${ }^{1}$ \\ sbenito@invi.uned.es}

\section{Almudena Agra-Gasqué ${ }^{2}$}

almudena.agragasque@educa.madrid.org

${ }^{1}$ Universidad Nacional de Educación a Distancia. España

${ }^{2}$ Consejería de Educación - Comunidad de Madrid, España

Fecha de recepción: 7/04/2020

Fecha de aprobación: 25/08/2020

\section{Resumen}

El presente artículo tiene como objeto analizar la trayectoria educativa del alumnado sordo en Espańa desde principios del siglo XX, en la que el papel de la lengua de signos como facilitador de la inclusión escolar toma cada vez más fuerza. Partimos de las necesidades comunicativo-lingüísticas de este alumnado justificando la importancia de la lengua oral y la lengua de signos. Comparamos los procesos de adquisición del lenguaje en contextos monolingües y bilingües. Después, describimos y valoramos el recorrido de los modelos educativos espańoles para dar una respuesta educativa ajustada al alumnado sordo, seńalando la presencia de ambas lenguas en cada uno. Destacamos las experiencias bilingües intermodales de la Comunidad de Madrid y hacemos referencia a proyectos semejantes de otras zonas del país. A modo de conclusión, se destaca el valor inclusivo de la lengua de signos y su utilidad para la actividad educativa, así como para el desarrollo social y emocional.

Palabras clave: lenguaje, lengua de signos, bilingüismo intermodal, inclusión, educación compartida.

\section{Abstract}

This article aims to analyze the educational trajectory of hearing impaired students in Spain since the beginning of the 20th century, in which the role of sign language as a facilitator of school inclusion takes more and more force. We start from the communicative-linguistic needs of these students, justifying the importance of oral language and sign language. We compare language acquisition processes in monolingual and bilingual contexts. Then, we describe and assess the journey of Spanish educational models to provide an educational response tailored to hearing impaired students, noting the presence of both languages in each. We highlight the intermodal bilingual experiences of the Community of Madrid and make reference to similar projects in other areas of the country. As a conclusion, the inclusive value of sign language and its usefulness for educational activity, as well as for social and emotional development, are highlighted.

Keywords: Language, sign language, cross-modal bilingualism, inclusion, co-enrollment. 


\section{Introducción}

Empezamos con un breve recorrido histórico a través de la educación de las personas sordas. Destacamos el papel de la LS (lengua de señas o signos), como código lingüístico de modalidad viso-gestual, principal vehículo de comunicación de muchas personas. Debemos aclarar que "señas" y "signos" son sinónimos con una historia diferente. En América Latina se generaliza la denominación "lengua de seńas" diferenciando del "signo lingüístico", referido a cualquier significante. Gascón (2003) explica que en Europa, entre los siglos XVI y XVIII, distinguían "seña" como forma natural de expresión, de "signo" como parte de sistemas creados artificialmente por educadores oyentes. Desde del siglo XIX se acepta la denominación "lengua de signos" como traducción literal de la palabra francesa. Cabeza e Iglesias (2015) refieren que la influencia actual es anglosajona, por mayor similitud visual con la expresión sign (langua$g e)$, que la forma patrimonial "seńa".

A continuación, exponemos la variabilidad de necesidades comunicativas del alumnado que justifican el uso de LS y otros sistemas de apoyo a la comunicación, a pesar del avance tecnológico. Al igual que se cuestiona la necesidad de la LS, la denominación "personas sordas" tampoco es aceptada de forma global. El término médico que indica pérdida de audición es "hipoacusia" y, en sentido estricto, solo se llama sordera a la hipoacusia en grado profundo. Algunas personas que siguen conversaciones en LO (lengua oral) utilizando medios técnicos prefieren hablar de "discapacidad o pérdida auditiva". Además, suelen sentirse de forma distinta a quienes utilizan LS. Y es que socialmente, igual que ocurre con las demás lenguas, se identifican como grupo con identidad social y cultural propia: la "comunidad sorda".

Proseguimos revisando investigaciones sobre la adquisición de un código lingüístico y comparamos el proceso en LO, en LS y en contextos bilingües. En este sentido, distinguimos bilingüismo LO-LS de bilingüismo castellano-inglés, muy presente en los centros educativos. Siguiendo a Morales (2010), un modelo bilingüe intermodal incluye dos lenguas con canales diferentes: una LS y una o más LO, mientras que un bilingüismo intramodal o unimodal se refiere a dos LO o a dos LS.

Tras esta fundamentación histórica y teórica, presentamos las opciones de escolarización implementadas en España desde principios del siglo XX hasta ahora, momento en que el modelo de educación inclusiva nos lleva al uso multipotencional de la LS. Desde nuestro punto de vista esta idea no es novedosa, pero no había un texto que la tratara de forma narrativa y cronológica y que pudiera servir de ejemplo a otros sistemas educativos, tanto en nuestros aciertos como en nuestras fallas. Por tanto, cerramos nuestro trabajo con una valoración comparativa de los modelos analizados.

Respecto a la terminología más reciente, en consonancia con los principios inclusivos, el concepto "diversidad funcional" empieza a sustituir a discapacidad, refiriéndose a una globalidad de personas con funcionamientos diversos sin poner el acento en la causa, sino en la necesidad de medidas inclusivas para responder a la natural heterogeneidad. No obstante, hablaremos en general de "sordos o sordas" desde el respeto a todas las personas. Primero, por economía lingüística, evitando expresiones contradictorias como "diversidad funcional auditiva", y, segundo, por subrayar el derecho a aprender y utilizar la LS, sin denostar, por supuesto, a la LO y escrita.

\section{La lengua de signos en la educación de las personas sordas: un breve recorrido histórico}

¿Lengua oral o lengua de señas/signos? Allá donde hay personas sordas, surge esta disyuntiva. A lo largo de la historia, encontramos posiciones educativas controvertidas sobre qué lengua utilizar o priorizar. Dichas posiciones se han alternado y simultaneado en el tiempo.

Sacks (1989) explica los "vaivenes" sufridos. Relata que durante los siglos XVI y XVII, el objetivo era que los sordos hablasen, pero no se consiguió. En el 
siglo XVIII, el abad De l'Epée, pedagogo y logopeda francés, utiliza un código de comunicación manual y se generaliza la defensa de la LS como lengua natural de las personas sordas. Pero los problemas comunicativos continuaron y, ante posturas encontradas, en 1880 en el Congreso Internacional de Milán se suprimió la educación en LS en toda Europa y disminuyó drásticamente el número de profesionales sordos. El objetivo fue la enseñanza de la LO para facilitar la comunicación. Esta "corriente oralista" continuó hasta 1980, cuando se celebró el Congreso Internacional de Hamburgo y se retomó la LS en contextos educativos. Fueron fundamentales las aportaciones lingüísticas sobre American Sign Language (Stokoe, 1960) que culminaron con el reconocimiento como lengua completa y estructura propia. A partir de los años 70, otros investigadores (Stewart, 1983; Akamasum \& Armour, 1987; Brodesky \& Cohen, 1988) demostraron que la LS transmite información abstracta y compleja, igual que la LO.

Otra importante fuente de investigación-acción es la Gallaudet University, escuela de gramática fundada en Washington en 1864 para niños sordos y ciegos. Actualmente es el único centro de Educación Superior oficialmente bilingüe intermodal.

En España, hasta los años 80, todo el alumnado con discapacidad asistía a CEE (centros de educación especial). En ese contexto, algunos eran específicos para sordos.

La actual Constitución española de 1978 reconoce los principios de normalización, integración e individualización. En 1985-1986 empieza la integración con carácter experimental, escolarizando en centros ordinarios (normalización) al alumnado con NEE (necesidades educativas especiales), que con adaptaciones curriculares y apoyos especializados (individualización) pudiera acercarse al nivel general. El alumnado que requería atención más intensiva y especializada permanecía en los CEE. Con la Ley de Ordenación General del Sistema Educativo de 1990, este sistema se generaliza. Y hacia principios de los años 90, junto con una mejor consideración de las LS, los CEE específicos para sordos se abren al alumnado oyente, surgiendo a partir de algunos de ellos los proyectos singulares de educación bilingüe intermodal. Fue una "auténtica revolución educativa".

Una década después, la principal crítica es que diferenciamos demasiado al alumnado con NEE dentro de los centros ordinarios. Se empieza a hablar de inclusión (Echeíta \& Verdugo, 2004).

Actualmente vivimos un nuevo proceso de cambio, ya que la Ley de Educación de 2006, modificada por la Ley para la Mejora de la Calidad Educativa de 2013, recoge el principio de inclusión.

Por otro lado, con la mejora de los medios técnicos para las personas sordas y las mayores posibilidades de acceso a ellos, se vuelve a cuestionar la necesidad de la LS.

Sin embargo, posteriormente queda aprobada la Ley $27 / 2007$, de 23 de octubre, por la que se reconocen las lenguas de signos espańolas [...], que recoge especialmente la LSE (LS española) y y la LSC (LS catalana). Y a nivel internacional, en 2016 se reconoce el derecho a la educación inclusiva en el Comité de Naciones Unidas sobre los Derechos de las Personas con Discapacidad, y se insta a tomar medidas para asegurar el aprendizaje de la LS y promover la identidad lingüística de la comunidad sorda.

\section{Alumnado sordo y variabilidad de necesidades comunicativo- lingüísticas}

El lenguaje es una capacidad que nos identifica como seres humanos eminentemente sociales. Monfort y Juárez (1987) lo definen como principal medio de comunicación y, en consecuencia, debemos aprenderlo en contacto con otras personas. Distinguen tres funciones principales:

- Función comunicativa del lenguaje, base de las relaciones interpersonales.

- Función de representación, que implica conceptualizar el mundo de forma "racional y económica” y relacionar ideas: razonamiento verbal. 
- Función reguladora de la personalidad y el comportamiento social: el lenguaje interviene en la clarificación de normas y límites, y más tarde sirve para expresar afectos y adaptar conductas sociales. Este lenguaje aparece de forma incidental y es "menos visible para las personas sordas", porque hay menos feedback informativo.

Debemos diferenciar "lenguaje" como capacidad humana de "lengua o código lingüístico" a utilizar, sea oral o signado; será vehículo de acceso a la comunicación, la información y la cultura, y un importante factor para la identificación con un grupo social.

En definitiva, es fundamental estructurar nuestra mente "construyendo lenguaje" lo antes posible, lo que justifica que cada persona lo haga con códigos lingüísticos que le resulten accesibles. Por este motivo, muchos autores coinciden en la conveniencia del aprendizaje temprano de la LS (Castro, 2002).

La variabilidad entre personas sordas implica diferencias en las condiciones necesarias para "construir lenguaje" y, en consecuencia, se podría decidir qué escolarización conviene a cada uno. Un contexto bilingüe intermodal es lo más enriquecedor, pero no siempre es imprescindible; dependerá de factores médicos, audiológicos, familiares, escolares y sociales como los siguientes:

- Grado y tipo de pérdida auditiva. Existencia de diagnósticos médicos añadidos.

- Detección temprana favorecerá su evolución.

- Prótesis apropiadas a las características médicas de su hipoacusia. No es imprescindible lo último en tecnología, sino lo preciso en cada caso, aunque no existen soluciones tecnológicas completas para todos.

- Medidas comunicativas, educativas y terapéuticas para favorecer su desarrollo.

- Tipo de estimulación lingüística en el contexto familiar y social.

Las personas sordas suelen compensar sus dificultades de forma parcial con gran esfuerzo, utilizando todas sus capacidades. Sus necesidades pueden pasar desapercibidas:

- Dificultades para acceder a la información y la comunicación.

- Desarrollo más lento o menor en LO y escrita.

- Pueden tener menor nivel académico o menos conocimientos del mundo.

- Menos experiencias sociales que influyen en su desarrollo socio-afectivo.

A nivel tecnológico hay que considerar el avance innegable de los implantes cocleares. Aunque los resultados no sean óptimos, han permitido acceder al lenguaje "vía auditiva" a personas sordas en grado profundo, que con otras prótesis no podrían conseguirlo. Tras la operación quirúrgica inicial, la persona debe hacer una terapia de reeducación logopédica para asociar sonidos con significados y acceder a la LO. Durante este tiempo, el uso de LS o sistemas de apoyo a la comunicación ayuda a "construir lenguaje". Las investigaciones arrojan resultados variables respecto a la conveniencia del uso de sistemas de comunicación viso-gestuales.

Destacamos el estudio longitudinal de Pérez et al. (2014) en cuatro centros de la Comunidad de Madrid con modalidad bilingüe intermodal. Investigan el desarrollo de vocabulario comprensivo y expresivo en alumnado con implante coclear y concluyen lo siguiente:

- La interacción en LO y LS favorece la evolución del vocabulario en ambas lenguas.

- Inicialmente adquieren más vocabulario en LS, pero pasados dos ańos con implante coclear desarrollan más vocabulario en LO. Ocurre en el momento en que implante y tratamiento logopédigo posterior hacen más eficaz su audición.

- La LS no dificulta el desarrollo de la LO y, además, todos los sordos de la muestra cuya lengua materna es la LS obtienen buenos 
resultados en LO, junto con un alto porcentaje que iniciaron el aprendizaje de la LS antes de cumplir 3 años.

\section{Adquisición de un código lingüístico}

A continuación, comparamos el proceso de adquisición del lenguaje cuando se da en LO, en LS o en ambas lenguas, en contextos bilingües.

\subsection{Adquisición de la lengua oral}

Los niños y niñas oyentes adquieren lenguaje oral mediante un proceso natural, como resultado de la interacción social. Sacks (1989) defiende que tenemos cierto potencial básico innato, pero necesitamos que lo active otra persona con competencia lingüística.

Sin embargo, a los sordos se les debe enseñar LO de forma adecuada e intencional, con bastante esfuerzo. Un bebé sordo está en desventaja para acceder a la información, lo que le llevará a perder el balbuceo temprano. Según estudios de Petitto (2000), hasta los 12 meses se produce balbuceo sin percepción de sonidos, debido a que la maduración lingüística de base neurológica posibilita el procesamiento de distintos tipos de señales, en este caso señales motoras. Posteriormente, si falta feedback auditivo, dejan de balbucear y la familia, al no ver cumplidas sus expectativas, puede interrumpir la interacción, afectando negativamente el desarrollo. Como consecuencia de la pérdida auditiva, la adquisición de la $\mathrm{LO}$ es más lenta.

\subsection{Adquisición de la lengua de signos}

La adquisición de la LS por parte del alumnado sordo goza de características y etapas similares a la adquisición de la LO por los oyentes. En iguales circunstancias de acceso sensorial, estimulación y exposición a las lenguas, sordos y oyentes tienen la misma posibilidad de adquirir una competencia lingüística adecuada.

Presentamos un patrón evolutivo a partir de resultados de varias investigaciones (Ciocci \& Baran, 1998;
Bonvillian \& Folven, 1993; Marschark, 2001):

- 12 meses:

- Primeros signos, de forma más temprana que en LO, porque la motricidad visomanual madura antes que la fonatoria.

- Repiten componentes de signos a modo de balbuceo manual.

- Primeros años:

- Adquieren aceleradamente signos referidos a objetos y acciones, potenciando su capacidad comunicativa.

- Aparecen gestos naturales con una función comunicativa potente como precedentes de signos.

- 3 años: modifican signos haciendo inflexiones, pero hasta los 4 años no toman conciencia de cómo cambia el significado.

- Entre 3 y 4 años: hacen extensivas reglas generales (hiperregularizaciones).

- 4 años:

- Modifican signos para añadir significados específicos (lugar o dirección).

- Utilizan intencionalmente la expresión facial para matizar significados.

- Describen sucesos y explican sus causas.

○ Transmiten deseos y preferencias.

- 5 años: cumplen las reglas lingüísticas de la LS.

- Entre 4 y 7 ańos: adquieren competencia para atender peticiones de clarificación de sus mensajes.

- De 7 a 15 años: usan las reglas discursivas propias de la lengua escrita y la LS. 


\subsection{Adquisición de lengua oral y lengua de signos: bilingüismo}

Las investigaciones realizadas en entornos educativos muestran

[...] al menos cuatro contextos diferentes: a) uso de signos como apoyo a la comunicación oral; b) uso de signos en forma de comunicación bimodal; c) uso de la LS en contextos de bilingüismo sucesivo (lengua de signos - lengua escrita), a menudo en situaciones de educación especial, y d) uso de la LS en enfoques educativos bilingües simultáneos en contextos de integración escolar (Pérez et al., 2014, p. 87).

Según Robles (2012), el bilingüismo no es la suma de dos lenguas, sino un continuo entre la competencia lingüística de ambas que dependerá también del contexto familiar y escolar. Destaca el derecho de cualquier persona sorda a "crecer bilingüe" desde la opción educativa bilingüe-bicultural.

Algunos niños sordos reciben en casa una combinación simultánea de LO y LS. Sus padres, normalmente oyentes, suelen utilizar el sistema bimodal, ya que la LS que emplean es una variedad de LO signada: mientras el vocabulario se toma de la LS, la sintaxis y el orden de las palabras corresponde a la LO, lo que permite la expresión simultánea de ambas. Schlesinger (1978) fue el primero en referirse a esta situación denominándola "bimodalismo" y diferenciándola del "bilingüismo”.

En familias sordas en las que la LS está presente desde el nacimiento, se observa mayor velocidad en el desarrollo comunicativo-lingüístico gracias también a la facilidad para adaptarse a hijos con necesidades comunicativas comunes.

El inicio temprano de la escolarización en contextos educativos con bilingüismo intermodal es buen momento para beneficiarse de la exposición e interacción en ambas lenguas, con independencia del entorno comunicativo familiar.

Hoff y Core (2015) explican que aprender una lengua supone menos tiempo que aprender dos simultáneamente y el desarrollo en cada lengua es más lento que el de una sola en los monolingües. Se pueden apreciar ligeros desfases en desarrollo gramatical y de vocabulario al evaluar cada lengua por separado, pero el nivel es semejante en habilidades fonológicas y narrativas. La evolución se distribuye de manera desigual entre ambas lenguas. Así, el concepto de "vocabulario conceptual" se refiere a la suma de términos que conocen en ambas lenguas - puntuación compuesta-, ya que pueden adquirir unos conceptos en una y otros en otra, y sería equivalente al vocabulario adquirido por personas monolingües.

Durante mucho tiempo el bilingüismo se consideraba negativo para el desarrollo lingüístico, especialmente cuando una de las lenguas tenía escaso reconocimiento social. Hoy se considera beneficioso tener competencia en varias lenguas y se conocen las ventajas cognitivas. Las personas bilingües presentan mejores habilidades en funciones ejecutivas por el control e inhibición lingüística que su cerebro realiza de forma continua.

Según Alcina-Madueño (2010), las investigaciones sobre experiencias educativas bilingües durante toda la Educación Primaria producen resultados académicos, lingüísticos y cognitivos positivos. Todo cambia cuando en un centro confluye alumnado muy diverso respecto al modelo comunicativo previo. Estas conclusiones procedentes de la investigación permiten tomar decisiones de política educativa.

\section{La escolarización de las personas sordas en España: en camino hacia modelos más inclusivos}

La historia de la escolarización del alumnado sordo es común en toda España hasta 1996, cuando las competencias educativas van pasando del Gobierno central a las comunidades autónomas. Después, cada comunidad fue desarrollando la normativa nacional y de ahí se derivan experiencias singulares diversas. Haremos un recorrido cronológico desde modelos iniciales y comunes hasta los más actuales e inclusivos. 


\subsection{Modelo dual: enseñanza general o especial}

Existen experiencias educativas adaptadas al alumnado sordo desde hace varios siglos. Pero el modelo educativo dual se sistematiza a principios del siglo XX. Alcina-Madueño (2014) detalla avatares políticos, económicos y educativos que condicionaron el proceso.

La Ley de Instrucción Pública de 1857 hacía obligatoria la educación elemental para todas las personas. Por eso, el Colegio Nacional de Sordomudos de Madrid, fundado en 1805, se fusionó con la Escuela Normal de Ciegos y pasó a gestionarse con fondos nacionales. La ley creaba una red de Colegios de Sordomudos y Ciegos, municipales o provinciales, en toda España. Hacia 1900 tenía presencia en Barcelona, Salamanca, La Coruña, Burgos, Zaragoza, Sevilla, Alicante, Valencia y Vizcaya. Paralelamente surgían centros privados, como los colegios de religiosas "La Purísima” en varias ciudades. Todos tenían residencia-internado. Con el tiempo incorporaron enseñanzas de formación profesional.

En el empeño por escolarizar a toda la población, intentaban compensar la falta de plazas públicas con mayor oferta en el Colegio Nacional, hasta 1985, aunque no hubiera becas para todos.

Entre 1910 y 1923 se institucionalizó la educación especial y desde 1933 se separan de nuevo las enseñanzas de sordomudos y ciegos. Se crean otros colegios para personas con discapacidad intelectual. La Guerra Civil española (1936-1939) y la Ley de Educación Primaria de 1945 suponen un retroceso respecto a oferta y calidad. Con la Ley General de Educación de 1970 se hace una reforma pedagógica que mejora la calidad, aumenta la obligatoriedad hasta los 14 años y consigue finalmente la generalización de la enseñanza a todas las personas.

Durante este largo periodo, las características han sido semejantes, a saber:

- Modelo educativo dual: enseñanzas generales o especiales. Las personas sordas acudían a ambos tipos de centros.
- Organización escolar de la enseñanza especial basada en grupos homogéneos.

- Currículo común en enseñanzas general y especial, con pequeńas adaptaciones en la segunda (excepto de 1945 a 1970, con bajos niveles de exigencia en enseñanza especial).

- Se insistía en la formación del profesorado y exigían una especialización para sordos y ciegos. Con el tiempo se creó una titulación diferenciada.

- El principal objetivo era oralizar al alumnado con métodos adecuados. Recordemos que la LS estuvo prohibida desde el Congreso de $\mathrm{Mi}$ lán de 1880 hasta un siglo después.

- Otro objetivo fue la formación profesional. El Colegio Nacional llegó a ofrecer formación para siete profesiones.

A partir de la renovación pedagógica de 1970, utilizaban metodologías tradicionales de enseñanza, con estrategias como las siguientes:

- Adaptaciones de acceso a la información: uso abundante de "ayudas lingüísticas visuales", como apoyarse en lectura labio-facial o en palabras escritas para clarificar el mensaje.

- Menor exigencia en producción escrita si en los exámenes se demostraban los conocimientos.

- Repetición como estrategia para interiorizar contenidos: prioridad de memoria sobre comprensión.

- Durante los últimos años, aunque de forma poco sistemática, empezaban a utilizar signos como apoyo a la LO o sistema bimodal.

Estaba próximo el fin de la prohibición de la LS y, tras siglos de educación especial más o menos institucionalizada, se avecinaban tiempos de grandes cambios. 


\subsection{Modelo de integración}

Este modelo llega con el Real Decreto 334/1985, de 6 de marzo, de ordenación de la Educación Especial, que pone fin al sistema paralelo y excluyente anterior. Los alumnos con NEE (necesidades educativas especiales) también son atendidos por la escuela ordinaria, con dotación de recursos personales y materiales. La integración implica una atención más normalizada a este alumnado, compartiendo currículo (adaptado), recursos y espacios comunes con el alumnado de modalidad ordinaria. No obstante, se mantienen los CEE para quienes requieren una atención intensiva y especializada durante todo el horario escolar.

Desde entonces, la mayor parte del alumnado sordo se escolariza en la modalidad de integración y dispone de lo siguiente:

- Profesorado especialista en Pedagogía Terapéutica y en Audición y Lenguaje, a veces competente en LSE:

- En colaboración con tutores y profesorado ordinario, contribuyen a hacer adaptaciones del currículo para que el alumnado con NEE pueda acercarse a los objetivos planteados con carácter general.

- Ambos perfiles profesionales realizan sesiones de apoyo dentro o fuera del aula, en pequeño grupo, para trabajar los elementos de cada adaptación curricular individual.

- El alumnado sordo necesitará habitualmente medidas de acceso a la comunicación e información, así como adaptaciones lingüísticas más o menos significativas.

- De forma excepcional, cuentan con personal no docente, a saber:

- Asesores sordos especialistas en LSE para enseñar la lengua, asesorar y apoyar al alumnado sordo, a sus familias y al profesorado. Son modelos adultos de identidad y referencia que facilitan el desarrollo socio-emocional.
- En Educación Secundaria, intérpretes de LSE para acceder a la información proporcionada en LO.

- Asimismo, tendrán seguimiento de profesionales de la red de orientación educativa.

Se abre la posibilidad de la adaptación curricular, lo cual supone un hito fundamental, pues se parte del currículo ordinario y se adapta individualmente a sus necesidades. La adaptación puede ser de eliminación de objetivos, cada vez más extinguida, frente a la reformulación, aplazamiento o exención parcial (por ejemplo, en inglés). Otro elemento sustancial es la inclusión del aprendizaje de LS como objetivo o contenido curricular. En este sentido, destacamos el actual Currículo de la Lengua de Signos Española para las etapas educativas de Infantil, Primaria y Secundaria (Esteban et al., 2017).

Sin embargo, en este modelo, la administración no exige requisitos específicos de formación en LSE al profesorado, lo que compromete la calidad educativa.

Por otro lado, cuanto más alumnado sordo vaya a un centro educativo, contará con más profesionales, mayor grado de especialización, sensibilidad y conocimiento de LS u otros sistemas y recursos de apoyo a la comunicación que acompañen a los medios técnicos y al cuidado de las condiciones del aula para facilitar la lectura labiofacial (Velasco \& Pérez, 2017). La elección de estos medios irá en función del momento y el objetivo de trabajo:

- LS como lengua vehicular del currículo.

- Sistema bimodal para aumentar el léxico y mejorar la percepción del orden de las palabras.

- Palabra complementada para visualizar y aclarar la labiolectura de los fonemas. De forma indirecta, mejora la competencia fonológica de la LO y favorece la lectoescritura.

- Múltiples visualizadores fonéticos o gestos recuerdo para trabajar la articulación.

- Abecedario dactilológico para crear representaciones gráficas manuales que, a su vez, supone un puente entre LS, LO y escrita. 
- Adaptaciones lingüísticas, entre ellas, adaptaciones de textos para facilitar el acceso a la información en otras áreas de conocimiento.

Estas circunstancias aconsejan la creación de centros ordinarios preferentes para alumnado sordo en los que rentabilizar recursos y socializarse junto con sordos y oyentes. El alumnado sordo podrá agruparse para recibir apoyo y conocer a iguales, lo que favorecerá los procesos de identidad como persona sorda (Valmaseda, 2015). Dicha opción implica la dotación de los recursos específicos de manera predominante en un centro por zona, de forma que acudan los alumnos con domicilio cercano. Algunos cuentan con transporte escolar.

Se han ido creado bastantes centros preferentes y, entre ellos, destacan por su mayor experiencia los antiguos CEE específicos para alumnado sordo. Actualmente están abiertos a todo el alumnado, y algunos han desarrollado proyectos singulares de educación bilingüe intermodal. Lo veremos con más detalle en el apartado 5.4 .

\subsection{En camino hacia la inclusión}

Tras comprobar que el modelo de integración no ha respondido a las expectativas iniciales, hacia 2004, la idea de inclusión frente a integración va tomando fuerza y al escolarizar al alumnado con NEE, la expresión "modalidad ordinaria con apoyos" sustituye a "modalidad de integración". La Ley de Educación de 2006 añade el principio de inclusión al de integración, y la Ley para la Mejora de la Calidad Educativa de 2013, que modifica a la anterior, elimina el principio de integración y habla solamente de inclusión. No obstante, en la práctica aún nos ubicamos en el modelo de integración, ya que la normativa no desarrolla un cambio estructural para atender al alumnado con NEE. En bastantes centros están reflexionando e implementando nuevas estrategias metodológicas que contribuyan a la inclusión educativa, pero aún queda mucho por hacer.

Booth et al. (1998) ya definían la inclusión como proceso de mejora de las instituciones educativas para eliminar las barreras que limitan presencia, aprendizaje y participación del alumnado en la vida de los centros educativos, con particular atención a aquellos más vulnerables. De ahí "las tres P" o principios inclusivos:

- Presencia de todo el alumnado con independencia de sus características.

- Participación real en actividades.

- Progreso y aprendizaje sin disminuir expectativas, potenciando las posibilidades reales de cada uno.

Muntaner (2019) nos da la clave para pasar de la lógica de la homogeneidad a la de la heterogeneidad:

- La homogeneidad pone el foco en la categorización y diferenciación del alumnado, mientras que la heterogeneidad toma la diversidad del alumnado como algo natural e incuestionable.

- Recursos especializados y ACI (adaptaciones curriculares individuales) se dirigen a que el alumnado con NEE se adapte a las exigencias globales: homogeneidad. Aceptar la heterogeneidad es una oportunidad para mejorar prácticas educativas y potenciar las capacidades individuales partiendo del conocimiento de la diversidad. Es decir, partir del currículo común para hacer una adaptación de aula, teniendo en cuenta todas las individualidades, no solo las del alumnado con NEE. Se atiende también a dificultades consideradas menores y al alumnado con más capacidad.

- El profesorado de apoyo trabaja cada ACI de manera diferenciada, muchas veces fuera del aula, para abordar objetivos más homogéneos con todo el grupo. La inclusión implica que el profesorado de apoyo se coordine y organice con el equipo docente, aportando conocimientos específicos, para trabajar de forma colaborativa y atender a todo el alumnado. En relación con las personas sordas, establecerán objetivos comunes de comunicación y lenguaje en la programación del aula. 
Según Espada et al. (2019), la inclusión implica adoptar medidas metodológicas y organizativas, así como elaborar propuestas de actividades que puedan ser resueltas en la misma aula, utilizando materiales diversos con distintos niveles de dificultad: diseño universal de aprendizaje. Esta organización requiere la presencia de varios profesionales en el aula. El número de profesionales especializados dependerá de la ratio de alumnado con NEE y determinará la organización de espacios y tiempos para articular las medidas inclusivas.

Por tanto, la "escuela para todos" es "una escuela de calidad" que requiere más profesorado, pero también más formación y creatividad. En este sentido, la creación de centros ordinarios preferentes para alumnado sordo no se contradice con la aplicación de medidas inclusivas que necesitarán el mantenimiento de los recursos ya mencionados. Las experiencias bilingües intermodales permitirán unificar esfuerzos, rentabilizar recursos y socializarse junto con iguales sordos y oyentes.

\subsection{Experiencias educativas de bilingüismo intermodal en la Comunidad de Madrid}

Actualmente cuatro centros educativos llevan a cabo experiencias de bilingüismo intermodal en la Comunidad de Madrid, aunque hay otros que combinan con eficacia LO y LSE sin responder a un modelo bilingüe.

Vamos a hablar de la Escuela Infantil Piruetas, el Colegio El Sol (antiguo Colegio Nacional), el Colegio Ponce de León y el Colegio Gaudem (antiguo Hispano-Americano de la Palabra). Aquí solo hacemos una instantánea del funcionamiento actual en Educación Infantil y Primaria, que se acerca bastante a la educación inclusiva. Para ello seguimos el trabajo de Pérez et al. (2019). Aunque hay algunas diferencias organizativas entre los cuatro centros, estas son sus características generales.

Empezamos explicando por qué hablar de "educación compartida" en lugar de "ordinaria con apoyos", puesto que les da su identidad como centros bilingües y es la clave fundamental para la inclusión.
Requieren dos profesionales por aula: uno es referente en LO (tutor o tutora) y otro en LSE (cotutor o cotutora, con formación como especialista en Audición y Lenguaje, que realiza funciones de apoyo dentro y fuera del aula). Sordos y oyentes siguen el mismo currículo a partir de un "input lingüístico dual", lo que posibilita el acceso a la información a través de una lengua o de ambas en función de características personales. La tarea del cotutor o cotutora no consiste en interpretar de forma exacta de LO a LSE, sino en hacer comprender la información, con explicaciones adicionales si es necesario. A veces utilizan sistemas de apoyo a la LO en función del objetivo del momento.

La tarea "compartida" por estas "parejas pedagógicas" requiere reflexión sobre la filosofía que subyace a este modelo de trabajo y coordinación sistemática para dinamizar el aula. Se trata de hacer un verdadero trabajo colaborativo (Muntaner, 2019).

Los profesionales sordos, especialistas en LSE, complementan el trabajo del profesorado enseñando su lengua materna, apoyando el aprendizaje y dando especial sentido al enfoque bilingüe-bicultural como modelos de identificación adultos. Como resultado, tanto el alumnado sordo como el oyente se siente parte de las actividades y cultura del centro.

Esta organización implica un mayor número de los profesionales mencionados. Para rentabilizar recursos dando una atención adecuada, se escolarizan cinco o seis alumnos sordos por aula, frente a la ratio habitual de otros centros educativos de uno a tres alumnos con NEE. Como el lector imaginará, a pesar de los recursos disponibles, dar una respuesta ajustada a este reto requiere medidas inclusivas, para lo que en cada centro, en mayor o menor medida, han optado por utilizar metodologías activas, a saber: aprendizaje basado en proyectos, trabajo cooperativo y estructuración del aula en zonas.

Ya para terminar, otra singularidad de estos colegios, a excepción de la Escuela Infantil, es que conservan algunas aulas específicas de educación especial para alumnado con graves dificultades de comunicación, sin presentar discapacidades asociadas, como ocurre 
en los demás CEE. Con la organización y recursos disponibles, es la fórmula encontrada para darles la oportunidad de una escolarización ajustada a sus necesidades y más cercana a la modalidad ordinaria con apoyos o "educación compartida", con cuyo alumnado realizan algunas actividades conjuntas.

\subsection{Otras experiencias de bilingüismo intermodal}

Existen proyectos bilingües intermodales en otros países (Holanda, Estados Unidos, Hong Kong) y comunidades españolas. De estas últimas, presentamos brevemente algunos ejemplos.

\section{Tabla 1. Proyectos bilingües bimodales en regiones españolas. Experiencias singulares}

\begin{tabular}{|c|c|c|}
\hline $\begin{array}{l}\text { Comunidad } \\
\text { autónoma }\end{array}$ & Centro y nivel & Proyecto \\
\hline Cataluña & $\begin{array}{l}\text { Escuela Municipal Tres Pins } \\
\text { Ed. Infantil y Primaria }\end{array}$ & $\begin{array}{l}\text { Proyecto bilingüe LSC-LOC. } \\
\text { En cada nivel hay un grupo de } 3 \text { a } 4 \text { alumnos sordos que com- } \\
\text { parten el currículo con sus compañeros, pero también trabajan en } \\
\text { agrupamientos con logopedas con contenidos curriculares conjun- } \\
\text { tos. Adquieren LS catalana y LO escrita y hablada. }\end{array}$ \\
\hline $\begin{array}{l}\text { Región de } \\
\text { Murcia }\end{array}$ & $\begin{array}{l}\text { IES Infante Don Juan Manuel } \\
\text { Ed. Secundaria y Bachillerato }\end{array}$ & $\begin{array}{l}\text { Proyecto ABC. } \\
\text { Se utiliza la LS con alumnos sordos para la impartición de distintas } \\
\text { materias y como materia propia. } \\
\text { Diseño de oferta de enseñanza de LS como primera lengua. }\end{array}$ \\
\hline $\begin{array}{l}\text { Comunidad } \\
\text { Valenciana }\end{array}$ & $\begin{array}{l}\text { Colegio IVAF - Luis Fortich } \\
\text { Ed. infantil y Primaria }\end{array}$ & $\begin{array}{l}\text { Centro de Integración Preferente para alumnos con deficiencia } \\
\text { auditiva. } \\
\text { Reserva de cupo en los grupos-clases para sordos. } \\
\text { Atención logopédica desde } 1 \text { año. } \\
\text { Recursos tecnológicos y personal especializado en comunicación, } \\
\text { LS y otros sistemas de comunicación. }\end{array}$ \\
\hline Andalucía & $\begin{array}{l}\text { CEIP Tartessos } \\
\text { Ed. Infantil y Primaria }\end{array}$ & $\begin{array}{l}\text { Proyecto bilingüe-bicultural sordo-oyente. } \\
\text { Materia en currículo del centro para sordos y oyentes sobre LS y } \\
\text { cultura sorda en } 6^{\circ} \text { E.P. } \\
1 \text { materia no lingüística en LS a sordos y oyentes. } \\
\text { Proyectos Bi-Bi: nativo de LSE en aula en impartición bilingüe en } \\
\text { materias no lingüísticas en } 3^{\circ} \text { E.P. }\end{array}$ \\
\hline País Vasco & $\begin{array}{l}\text { CEP Basurto } \\
\text { Ed. Infantil y Primaria }\end{array}$ & $\begin{array}{l}\text { Proyecto bilingüe-bimodal. } \\
\text { Alumnos sordos en grupos de referencia con profesorado bilingüe } \\
\text { (en LO y LS). } \\
\text { Logopedia específica para sordos. }\end{array}$ \\
\hline Galicia & $\begin{array}{l}\text { CEIP Escultor Acuña } \\
\text { Ed. Infantil y Primaria }\end{array}$ & $\begin{array}{l}\text { Proyecto de impartición de materia de LS. } \\
\text { Aprendizaje para todo el alumnado sordo y oyente. } \\
\text { Profesores de audición y lenguaje. } \\
\text { Intérpretes de LS en impartición de materias. }\end{array}$ \\
\hline
\end{tabular}

Nota: Elaborado a partir de la web https://www.cnlse.es/es/resources/3/31/ccaa/65. 
Podemos apreciar una alta coincidencia en la presencia de LS como objeto o material curricular, incluso para el alumnado oyente y como apoyo en rehabilitación logopédica. Se potencia LS y LO, favoreciendo la inclusión e interacción. Al ser ambas lenguas vehiculares para impartir y adquirir el currículo, son menos necesarias las adaptaciones o el apoyo fuera del aula, es decir, facilita la inclusión.

\section{Valoración de los cambios producidos en la educación de las personas sordas desde 1970}

La transición entre modelos educativos conlleva modificaciones más y menos positivas. Para sintetizar las diferencias entre el modelo dual (educación ordinaria o especial) y el modelo de integración-inclusión (en su continuo evolutivo), hemos elaborado una tabla comparativa. Los datos provienen de la revisión bibliográfica y de documentos internos de la Comunidad de Madrid.

Hemos obviado lo relativo a personas sordas escolarizadas en centros ordinarios (1970-1990) por asemejarse a la situación actual de personas sordas que van a centros ordinarios no preferentes.

Tengamos en cuenta que los resultados educativos dependen del modelo o centro escolar, pero también de los cambios globales producidos durante estos cincuenta ańos:

- La evolución tecnológica ha facilitado un mejor "funcionamiento" de la vía auditiva a muchos alumnos.

- Existen nuevas estrategias para enseñar LO y escrita. Se prioriza la comprensión de contenidos sobre la memoria.

- El punto de partida de cada persona sorda cuando comienza su escolarización es más diverso. El alumnado de centros ordinarios y preferentes no bilingües suele tener capacidad para desarrollar la LO por "vía auditiva". Los centros bilingües atienden una mayor variabilidad de alumnado sordo, con ventajas respecto a la oferta educativa, pero con diferencias en los resultados.

Tabla 2. Comparación de modelos educativos para alumnado sordo en España (1970-2020)

\begin{tabular}{|c|c|c|c|c|}
\hline \multirow[b]{2}{*}{$\begin{array}{l}\text { Aspecto a } \\
\text { describir }\end{array}$} & \multirow{2}{*}{$\begin{array}{c}\text { MODELO DUAL: general o } \\
\text { especial } \\
(1970-1990)\end{array}$} & \multicolumn{3}{|c|}{ INTEGRACIÓN-INCLUSIÓN (1990-2020) } \\
\hline & & $\begin{array}{c}\text { Centros ordinarios y } \\
\text { preferentes }\end{array}$ & $\begin{array}{l}\text { Centros preferentes } \\
\text { singulares con bilin- } \\
\text { güismo intermodal }\end{array}$ & $\begin{array}{l}\text { CEE o AEE (aulas de edu- } \\
\text { cación especial de cen- } \\
\text { tros bilingües) }\end{array}$ \\
\hline $\begin{array}{c}\text { Centros } \\
\text { educativos }\end{array}$ & $\begin{array}{l}\text { - Centros ordinarios (sin } \\
\text { adaptaciones). } \\
\text { - CEE: alumnado con disca- } \\
\text { pacidad. } \\
\text { - CEE específicos adaptados } \\
\text { para "sordomudos" sin dis- } \\
\text { capacidades asociadas. }\end{array}$ & \multicolumn{3}{|c|}{$\begin{array}{l}\text { - Centros ordinarios (con adaptaciones). } \\
\text { - Centros ordinarios preferentes para alumnado con discapacidad auditiva: } \\
\text { - Cualquier centro de la zona que empieza a especializarse. } \\
\text { - Transformación de los antiguos CEE específicos para sordos: algunos } \\
\text { con bilingüismo intermodal. } \\
\text { - CEE para todas las discapacidades. } \\
\text { - Aulas de educación especial en centros ordinarios preferentes con bilin- } \\
\text { güismo intermodal. }\end{array}$} \\
\hline $\begin{array}{l}\text { Lenguas y } \\
\text { sistemas } \\
\text { de apoyo }\end{array}$ & $\begin{array}{l}\text { - LO: mayoritaria. } \\
\text { - Sistema bimodal: uso pro- } \\
\text { gresivo. } \\
\text { - LSE: comunicación fuera } \\
\text { del aula (aprendizaje infor- } \\
\text { mal). }\end{array}$ & $\begin{array}{l}\text { - LO: mayoritaria. } \\
\text { - Sistemas de apoyo } \\
\text { a LO: uso parcial. } \\
\text { - LSE: ocasional. }\end{array}$ & $\begin{array}{l}\text { - LO y LSE: habitual. } \\
\text { - Sistemas de apoyo } \\
\text { a LO: habitual se- } \\
\text { gún objetivos. } \\
\text { - LSE: aprendizaje } \\
\text { formal e informal. }\end{array}$ & $\begin{array}{l}\text { CEE: } \\
\text { - LO: mayoritaria. } \\
\text { - LSE y sistemas de apoyo } \\
\text { a LO: uso parcial. } \\
\text { AEE: } \\
\text { - LSE: mayoritaria. Apren- } \\
\text { dizaje formal e informal. } \\
\text { - Sistema bimodal y LO: } \\
\text { habitual. } \\
\text { - Otros sistemas de apoyo } \\
\text { a LO: habitual. }\end{array}$ \\
\hline
\end{tabular}


Revista Caribeña de Investigación Educativa | 2021, 5(1), 116-132

\begin{tabular}{|c|c|c|c|c|}
\hline \multirow[b]{2}{*}{$\begin{array}{l}\text { Aspecto a } \\
\text { describir }\end{array}$} & \multirow{2}{*}{$\begin{array}{c}\text { MODELO DUAL: general o } \\
\text { especial } \\
(1970-1990)\end{array}$} & \multicolumn{3}{|c|}{ INTEGRACIÓN-INCLUSIÓN (1990-2020) } \\
\hline & & $\begin{array}{l}\text { Centros ordinarios y } \\
\text { preferentes }\end{array}$ & $\begin{array}{l}\text { Centros preferentes } \\
\text { singulares con bilin- } \\
\text { güismo intermodal }\end{array}$ & $\begin{array}{l}\text { CEE o AEE (aulas de edu- } \\
\text { cación especial de cen- } \\
\text { tros bilingües) }\end{array}$ \\
\hline $\begin{array}{l}\text { AS (alum- } \\
\text { nos y alum- } \\
\text { nas sordos) }\end{array}$ & $\begin{array}{l}\text { - Centros ordinarios: minoría } \\
\text { AS. } \\
\text { - CEE: } 100 \% \text { AS. }\end{array}$ & $\begin{array}{ll}\text { - } & \text { 1-2 AS/aula. } \\
\text { - } & 0-2 \text { AS/centro ordi- } \\
& \text { nario. } \\
\text { - } & 1-20 \text { AS/centro } \\
& \text { preferente. } \\
\text { - } & 75 \% \text { AS de la Co- } \\
& \text { munidad de Madrid } \\
& \text { repartidos en multi- } \\
& \text { tud de centros. }\end{array}$ & $\begin{array}{l}\text { - } 5-6 \text { AS/aula (equi- } \\
\text { librio recursos/res- } \\
\text { puesta educativa). } \\
\text { - } 10 \text { AS en la Escuela } \\
\text { Infantil. } \\
\text { - } 75-100 \text { AS/colegio. } \\
\text { - } 25 \% \text { AS de la Co- } \\
\text { munidad de Madrid } \\
\text { entre los } 4 \text { centros. }\end{array}$ & $\begin{array}{l}\text { CEE: } \\
\text { - Minoría entre otras dis- } \\
\text { capacidades. } \\
\text { AEE: } \\
\text { - Mayoría entre otras dis- } \\
\text { capacidades. } \\
\text { - } 75-100 \text { AS/colegio }\end{array}$ \\
\hline \multirow[t]{2}{*}{$\begin{array}{l}\text { Desarrollo } \\
\text { lingüístico }\end{array}$} & \multirow{2}{*}{$\begin{array}{l}\text { Desarrollo lingüístico a me- } \\
\text { jorar: } \\
\text { - LO y escrita: desarrollo } \\
\text { variable con frecuentes e } \\
\text { importantes dificultades. } \\
\text { - LSE: buen nivel. }\end{array}$} & \multicolumn{2}{|c|}{$\begin{array}{l}\text { Desarrollo lingüístico aceptable (influido por } \\
\text { avances tecnológicos y nuevas metodologías): }\end{array}$} & $\begin{array}{l}\text { Desarrollo lingüístico a me- } \\
\text { jorar: }\end{array}$ \\
\hline & & $\begin{array}{l}\text { - LO y escrita: nivel } \\
\text { medio-bajo (variabi- } \\
\text { lidad). } \\
\text { - LSE: escaso o nulo } \\
\text { conocimiento. }\end{array}$ & $\begin{array}{l}\text { - LO y escrita: nivel } \\
\text { medio-bajo (gran } \\
\text { variabilidad). } \\
\text { - LSE: nivel variable } \\
\text { según preferencia } \\
\text { entre ambas len- } \\
\text { guas. }\end{array}$ & $\begin{array}{l}\text { CEE (alumnado sordo con } \\
\text { discapacidad intelectual): } \\
\text { - LO y escrita: nivel muy } \\
\text { bajo. } \\
\text { - LSE: escaso conocimien- } \\
\text { to. } \\
\text { AEE (suelen ser solo } \\
\text { sordos, gran dificultad } \\
\text { comunicativa, currículo } \\
\text { más básico): } \\
\text { - LO y escrita: desarrollo } \\
\text { variable con frecuentes } \\
\text { dificultades. } \\
\text { - LSE: buen nivel. }\end{array}$ \\
\hline $\begin{array}{l}\text { Desarro- } \\
\text { Ilo so- } \\
\text { cio-afec- } \\
\text { tivo }\end{array}$ & $\begin{array}{l}\text { - Sensación de normalidad } \\
\text { al margen de la sociedad } \\
\text { oyente. } \\
\text { - Escolarización con iguales } \\
\text { sordos que fortalece proce- } \\
\text { sos de identidad y autoesti- } \\
\text { ma. } \\
\text { - Fuerte sentimiento de } \\
\text { unión: "comunidad sorda". } \\
\text { - Comunicación mayoritaria } \\
\text { con personas sordas u } \\
\text { oyentes que conocen LSE. }\end{array}$ & $\begin{array}{l}\text { - Percepción de sus } \\
\text { dificultades en con- } \\
\text { tacto con compañe- } \\
\text { ros oyentes. } \\
\text { - Procesos de iden- } \\
\text { tidad y autoestima } \\
\text { complejos. } \\
\text { - Mayor o menor } \\
\text { comunicación con } \\
\text { iguales oyentes } \\
\text { según nivel auditivo } \\
\text { y lingüístico. } \\
\text { - Puede haber sen- } \\
\text { sación de soledad y } \\
\text { dificultad para rela- } \\
\text { cionarse. }\end{array}$ & $\begin{array}{l}\text { - Percepción más re- } \\
\text { alista y normalizada } \\
\text { de sus dificultades } \\
\text { en contacto con } \\
\text { compañeros sordos } \\
\text { y oyentes. } \\
\text { - Procesos de iden- } \\
\text { tidad y autoestima } \\
\text { más sencillos. } \\
\text { - Comunicación con } \\
\text { iguales sordos y } \\
\text { oyentes, con ten- } \\
\text { dencia a mayor } \\
\text { contacto con los } \\
\text { primeros. } \\
\text { - Sensación variable } \\
\text { de pertenencia a la } \\
\text { "comunidad sorda" } \\
\text { (mayor en hijos de } \\
\text { familias sordas). }\end{array}$ & $\begin{array}{l}\text { CEE: } \\
\text { - Percepción variable de } \\
\text { sus propias dificultades. } \\
\text { - Procesos de identidad y } \\
\text { autoestima complejos. } \\
\text { - Comunicación pobre con } \\
\text { iguales. } \\
\text { AEE: } \\
\text { - Percepción intensa de } \\
\text { sus dificultades. } \\
\text { - Procesos de identidad } \\
\text { y autoestima facilitados } \\
\text { por la fuerte cohesión del } \\
\text { grupo-clase. } \\
\text { - Sensación variable de } \\
\text { pertenencia a la "comuni- } \\
\text { dad sorda". } \\
\text { - Comunicación mayorita- } \\
\text { ria con personas sordas } \\
\text { u oyentes que conocen } \\
\text { LSE. }\end{array}$ \\
\hline
\end{tabular}


BENITO-MORENO • AGRA-GASQUÉ

La lengua de signos como recurso inclusivo en el sistema educativo español

\begin{tabular}{|c|c|c|c|}
\hline \multirow[b]{2}{*}{$\begin{array}{l}\text { Aspecto a } \\
\text { describir }\end{array}$} & \multirow{2}{*}{$\begin{array}{c}\text { MODELO DUAL: general o } \\
\text { especial } \\
(1970-1990)\end{array}$} & \multicolumn{2}{|c|}{ INTEGRACIÓN-INCLUSIÓN (1990-2020) } \\
\hline & & $\begin{array}{c}\text { Centros ordinarios y } \\
\text { preferentes }\end{array}$ & $\begin{array}{cc}\text { Centros preferentes } & \text { CEE o AEE (aulas de edu- } \\
\text { singulares con bilin- } & \text { cación especial de cen- } \\
\text { güismo intermodal } & \text { tros bilingües) }\end{array}$ \\
\hline $\begin{array}{l}\text { Percep- } \\
\text { ción de } \\
\text { sus limita- } \\
\text { ciones por } \\
\text { la socie- } \\
\text { dad }\end{array}$ & $\begin{array}{l}\text { - Muchas limitaciones co- } \\
\text { municativas y para la vida } \\
\text { diaria. } \\
\text { - } \text { Creencias erróneas: "no } \\
\text { pueden hablar", discapaci- } \\
\text { dad intelectual añadida. }\end{array}$ & $\begin{array}{l}\text { - Limitaciones comunic } \\
\text { - Capacidad para las a } \\
\text { - Capacidad intelectua } \\
\text { - Creencia errónea difí } \\
\text { den hablar". } \\
\text { - Percepción de LSE: } \\
\text { dad. Algunas familias }\end{array}$ & $\begin{array}{l}\text { ativas variables. } \\
\text { ctividades de la vida diaria. Menor en CEE. } \\
\text { normal, salvo en CEE. } \\
\text { cil de cambiar: "algunos son sordomudos y otros pue- } \\
\text { osibilidad comunicativa o evidencia de su discapaci- } \\
\text { solo quieren LO. }\end{array}$ \\
\hline $\begin{array}{c}\text { Oferta } \\
\text { educativa } \\
\text { adaptada }\end{array}$ & $\begin{array}{l}\text { - } \\
\text { Educación Infantil (5-6 } \\
\text { años). } \\
\text { - Educación General Básica } \\
\text { (6-14 años) para todas las } \\
\text { personas sordas. } \\
\text { - } \text { FP (formación profesional): } \\
\text { hasta } 7 \text { profesiones. }\end{array}$ & $\begin{array}{l}\text { - Se prioriza Educació } \\
\text { - Educación Secundar } \\
\text { tación. } \\
\text { - "Reconversión" de lo } \\
\text { FP adaptada. } \\
\text { - Poca oferta educativ } \\
\text { pocos centros de est } \\
\text { los solicitantes). } \\
\text { - AEE como respuesta } \\
\text { resto de centros, per }\end{array}$ & $\begin{array}{l}\text { Infantil y Primaria (0-12 años). } \\
\text { a obligatoria (12-16 años) con menor grado de adap- } \\
\text { antiguos CEE que supuso eliminar mucha oferta de } \\
\text { bilingüe (aunque se escolaricen 5-6 AS/aula, hay } \\
\text { as características y no siempre hay plaza para todos } \\
\text { a alumnado con dificultad para escolarizarse en el } \\
\text { con respuesta más inclusiva que en los CEE. }\end{array}$ \\
\hline $\begin{array}{l}\text { Desarrollo } \\
\text { normativo }\end{array}$ & $\begin{array}{l}\text { - La ley permitía la escolari- } \\
\text { zación en centros ordina- } \\
\text { rios y especiales a elección } \\
\text { de la familia. }\end{array}$ & $\begin{array}{l}\text { - Elección familiar de c } \\
\text { - Normativa más inclus } \\
\text { o Plazas reservadas } \\
\text { dalidad "ordinaria } \\
\text { o CEE solo para alu } \\
\text { cial). } \\
\text { - En espera del desarr } \\
\text { Educación. } \\
\text { - Experiencias inclusiv } \\
\text { ferentes o no). Impor } \\
\text { mucho camino pendi } \\
\text { - Se crean los centros } \\
\text { nes de los centros bil } \\
\text { o Dificultad para ma } \\
\text { cesarios. } \\
\text { o No se asegura la } \\
\text { do con NEE. } \\
\text { o No se exige forma }\end{array}$ & $\begin{array}{l}\text { entro, pero no de la modalidad de escolarización. } \\
\text { iva: integración como paso intermedio: } \\
\text { para alumnado con NEE en centros ordinarios (mo- } \\
\text { son apoyos"). } \\
\text { nnado con mayores dificultades (modalidad espe- } \\
\text { ollo normativo para la inclusión, reflejada en la Ley de } \\
\text { as a nivel metodológico en centros ordinarios (pre- } \\
\text { ante esfuerzo de los centros bilingües LO-LS, con } \\
\text { pre de recorrer. } \\
\text { ingües intermodales: } \\
\text { tener la ratio de profesionales sordos y oyentes ne- } \\
\text { referencia alumnado sordo sobre el resto de alumna- } \\
\text { ción en LSE: influencia en la calidad. }\end{array}$ \\
\hline
\end{tabular}

Nota: Datos numéricos internos de la Comunidad de Madrid (2019). 


\section{Conclusiones}

Tras un siglo de experiencias educativas más sistemáticas con personas sordas, estamos viviendo momentos de cambio hacia modelos más inclusivos en los que la LS tiene un papel preponderante. Aunque los centros con bilingüismo intermodal ofrecen respuestas más amplias, debemos superar planteamientos absolutos puesto que la heterogeneidad de este colectivo implica distintas posibilidades educativas.

Fernández y García (2017) exponen los desafíos a los que se enfrentan los sistemas educativos iberoamericanos en materia de inclusión con población muy heterogénea a nivel social y de capacidades. Este artículo puede suponer una aportación valiosa para diseñar actuaciones educativas dirigidas a la inclusión del alumnado sordo en otros sistemas educativos, garantizando el principio de equidad frente a sus compañeros oyentes.

\section{Referencias}

Alcina-Madueño, A. (2010). Las lenguas de signos en la educación bilingüe: un enfoque plurilingüe e intercultural. Avances en Supervisión Educativa, (13). https://bit.ly/3aASAxa

Alcina-Madueño, A., \& Fajardo, O. N. (2014). La politica educativa de las enseñanzas de sordomudos en España a través del Colegio Nacional de Sordomudos de Madrid (1875-2000). [Disertación doctoral, UNED. Universidad Nacional de Educación a Distancia]. https://bit.ly/318N4Xz

Bonvillian, J. D., \& Folven, J. R. (1993). Sign language acquisition: developmental aspects. En $\mathrm{M}$. Marschark, M. D. Clark, et al. (Eds.), Psychological Perspectives on Deafness (pp. 229-265). Hillsdale, NJ: Lawrence Erlbaum Associates.

Booth, T., Ainscow, M., \& Dyson, A. (1998). England: inclusion and exclusion in a competitive system. En Booth, T. \& Ainscow, M. (Eds.), From then to $U_{s:}$ An international study of inclusion in education (pp. 193-225). London: Routledge

Brodesky, R., \& Cohen, H. (1988). The American Sign Language / English Studies Project: A Progress
Report. American Annals of the Deaf, 133(5), 33-35. http://dx.doi.org/10.1353/aad.2012.0685

Cabeza, C., \& Iglesias, S. (2015). Spanish Sign Language. En J. Bakken Jepsen, G. De Clerck, S. Lutalo-Kiingi y W. B. McGregor (Eds.), Sign Languages of the World. A Comparative Handbook (pp. 729-769). Ishara Press.

Castro, P. J. (2002). Aprendizaje del lenguaje en niños sordos: fundamentos para la adquisición temprana de lenguaje de señas. Temas de Educación, 19. https://bit.ly/3dVpd9Y

Ciocci, S. R., \& Baran, J. A. (1998). The use of conversational repair strategies for children who are deaf. American Annals of the Deaf, 143, 235-245. http://dx.doi.org/10.1353/aad.2012.0129

Constitución Española de 1978. Boletín Oficial del Estado, 311. https://bit.ly/3j9K6js

Echeíta, G., \& Verdugo, M. Á. (2004). La Declaración de Salamanca sobre necesidades educativas especiales 10 años después. Valoración y prospectiva. Universidad de Salamanca: Publicaciones del INICO.

Espada, R. M., Gallego, M. B., \& González-Montesino, R. H. (2019). Universal Design of Learning and inclusion in Basic Education. Alteridad. Revista de Educación, 14(2), 207-218.

http://dx.doi.org/10.17163/alt.v14n2.2019.05

Esteban, M. L., Aroca, E., Rodríguez, M., \& Sánchez, D. (2017). Currículo de la Lengua de Signos Española para las etapas educativas de Infantil, Primaria y Secundaria. Madrid: Real Patronato sobre Discapacidad.

Fernández, M. T., \& García, B. M. (2017). La educación inclusiva intercultural en Latinoamérica. Análisis legislativo. Revista de Educación Inclusiva, 9(2bis). https://bit.ly/2V8piPd

Gascón, A. (2003). ¿Señas o signos?: Evolución histórica. https://bit.ly/39RQCXb

Hoff, E., \& Core, C. (2015). What Clinicians Need to Know about Bilingual Development. Seminars in Speech and Language, 36(2), 89-99.

https://bit.ly/3c2WJto 
Ley de 9 de septiembre de 1857, de Instrucción Pública. Gaceta de Madrid, (1660), 9.

https://bit.ly/2QjD6En

Ley de 17 de julio de 1945, de Educación Primaria. Boletín Oficial del Estado, 199. https://bit.ly/31lWiri

Ley 14/1970, de 4 de agosto, General de Educación y Financiamiento de la Reforma Educativa (LGE). Boletin Oficial del Estado, 187. https://bit.ly/3gq1GOC

Ley Orgánica 1/1990, de 3 de octubre, de Ordenación General del Sistema Educativo (LOGSE). Boletin Oficial del Estado, 238. https://bit.ly/3gqh0KU

Ley Orgánica 2/2006, de 3 de mayo, de Educación, modificada por la Ley Orgánica 8/2013, de 9 de diciembre, para la Mejora de la Calidad Educativa (LOMCE). Boletín Oficial del Estado, 295.

https://bit.ly/2EkzCPE

Ley 27/2007, de 23 de octubre, por la que se reconocen las lenguas de signos españolas y se regulan los medios de apoyo a la comunicación oral de las personas sordas, con discapacidad auditiva y sordociegas. Boletin Oficial del Estado, 255.

https://bit.ly/2EwJoOc

Marschark, M. (2001). Language development in children who are deaf: A research synthesis [Versión electrónica]. Project FORUM. National association of state director of special education.

https://bit.ly/2yHwJFr

Monfort, M., \& Juárez, A. (1987). El niño que habla. Madrid: CEPE.

Morales, E. (2010). Características generales del bilingüismo inter-modal (lengua de signos/lengua oral). En: Martí i Castells, J., \& Mestres i Serra, J. M. (Eds.), Les llengües de signes com a llengües minoritàries: perspectivas lingüistiques, socials $i$ politiques. Actes del seminari del CUIMPB-CEI 2008 (pp. 175-188). Barcelona: Institut d'Estudis Catalans.

https://bit.ly/34iq1Br

Muntaner, J. J. (2019). Consideraciones para la intervención de los apoyos de la comunicación y el lenguaje en la escuela inclusiva. Revista de Logopedia, Foniatria y Audiología, 39(1), 41-48. https://doi.org/10.1016/j.rlfa.2018.06.004

Pérez, M., Valmaseda, M., de la Fuente, B., Montero, I., \& Mostaert, S. (2014). Desarrollo del vocabulario temprano en nińos con implante coclear escolarizados en centros con bilingüismo oral-signado. Revista de Logopedia, Foniatría y Audiología, 34(2), 85-97. https://doi.org/10.1016/j.rlfa.2013.07.008

Pérez, M., de la Fuente, B., Alonso, P., \& Echeíta, G. (2019). Four Co-Enrollment Programs in Madrid: Differences and Similarities. Co-Enrollment in Deaf Education (Oxford University Press), 235-256.

http://dx.doi.org/10.1093/oso/9780190912994.003.0012

Petitto, L. A. (2000). The acquisition of natural signed languages: lessons in the nature of human language and its biological foundation. En C. Chamberlain, J. P. Morford \& R. Mayberry (Eds.), Acquisition of Language by Eyes (pp. 41-50). London: Lawrence Erlbaum Associates.

Real Decreto 334/1985, de 6 de marzo, de ordenación de la Educación Especial. Boletín Oficial del Estado, 55. https://bit.ly/2FT5BXF

Robles, M. (2012). Tendencia educativa bilingüe y bicultural para la educación del sordo: Un nuevo camino hacia la inclusión. Unirevista.es, (1), 76-86. https://bit.ly/3bZHWiO

Sacks, O. (1989). Veo una voz. Madrid: Anaya.

Schlesinger, H. (1978). The acquisition of bimodal language. Sign Language of the Deaf, IM Schlesinger \& Namir eds. New York: Academic Press.

https://doi.org/10.1016/B978-0-12-625150-0.50008-3

Stewart, D. (1983). The use of signs by deaf children: the opinions of a Deaf Community. American Annals of the Deaf, 128(7), 878-883.

http://dx.doi.org/10.1353/aad.2012.0868

Stokoe, W. C. (1960). Sign language structure: An outline of the communicative systems of the American deaf. Silver Spring, MD: Linstock Press. 
Revista Caribeña de Investigación Educativa | 2021, 5(1), 116-132

Valmaseda, M. (2015). La alfabetización emocional de los alumnos sordos. Revista Latinoamericana de Educación Inclusiva, 150. https://bit.ly/3ggWoEO
Velasco, C., \& Pérez, I. (2017). Sistemas y recursos de apoyo a la comunicación y al lenguaje de los alumnos sordos. Revista Latinoamericana de Educación Inclusiva, 3(1), 77-93. https://bit.ly/2Rz2abJ

\section{CÓMO CITAR:}

Benito-Moreno, S. C., \& Agra-Gasqué, A. (2021). Lengua de signos como recurso inclusivo en el sistema educativo español. RECIE. Revista Caribeña de Investigación Educativa, 5(1), 116-132. https://doi.org/10.32541/recie.2021.v5i1.pp116-132 